Condensed Matter Physics, 1999, Vol. 2, No. 1(17), p. 37-51

\title{
Calculation of transport coefficients dispersion and a dynamic structure factor of simple liquid
}

\author{
V.V.Ignatyuk \\ Institute for Condensed Matter Physics \\ of the National Academy of Sciences of Ukraine, \\ 1 Svientsitskii Str., 290011 Lviv, Ukraine
}

Received October 15, 1998

\begin{abstract}
The dynamic structure factor and the dispersion of transport coefficients are calculated for a simple liquid on the basis of the Fokker-Planck equation for a nonequilibrium distribution function of collective variables. The mode-coupling theory is used to obtain a closed chain of equations for wave-vector- $(\boldsymbol{k})$ and frequency- $(\omega)$ dependent transport coefficients. It is shown that in the intermediate region of $k$ the relations of nonlocal thermodynamics give more reliable results, while in the case of small wavenumber values one could restrict oneself by local thermodynamics. An analysis of the sound mode is performed and the coefficient at $k^{5 / 2}$ for a sound frequency dispersion is calculated. The obtained results are compared with both the experimental data and computer simulation.
\end{abstract}

Key words: Fokker-Planck equation, time correlation functions, nonlinear fluctuations, mode-coupling theory

PACS: $05.60 .+w, 05.70 . \mathrm{Ln}, 51.10 .+y, 61.20 . \mathrm{Lc}, 61.20 . \mathrm{Ne}$

\section{Introduction}

The theoretical investigation of nonlinear fluctuations appears to be an extremely complicated, interesting and completely unstudied problem in modern statistical mechanics. This problem has, besides a purely theoretical point of view, an important applied aspect as well. It is clear that permanent attention has been paid to the investigation of nonlinear fluctuations in different objects: dense gases and plasmas $[1,2]$, simple and ionic liquids [3-7], disordered [8] and quantum systems [9], liquid-glass phase transitions [10]. One has obtained nonlocal transfer equations, calculated transport coefficients, carried out the analysis of structural properties of the systems, the investigation of time correlation functions with consideration of a self-energy operator.

The result of the influence of fluctuations is the appearance of "long tails" 
in time correlation functions which exhibit nonexponential damping in time. Besides, the transport coefficients appear to be dependent on both wave-vector $\boldsymbol{k}$ and frequency $\omega$. The appearance of the mentioned dispersion for transport coefficients is observed in neutron scattering experiments and is characterized by a sound frequency renormalization $[3,11]$ that leads to a shift of the Brillouin peak of the dynamic structure factor. A typical feature of the dispersion in transport coefficients is its nonanalytical dependence on $\boldsymbol{k}$ and $\omega$ [12]. There are several methods for obtaining these nonanalyticities, namely, the Green functions method [13], the formalism of the Fokker-Planck equation (FPE) for the distribution function of coarse-grained dynamic variables [14,15], the projection technique on the basis of nonlinear fluctuations [16]. In each mentioned case the procedure of the decoupling of higher-order time correlation functions in products of pair time correlation functions is used, allowing one to obtain a closed chain of equations for the determination of a self-energy matrix and, consequently, for the calculation of $\boldsymbol{k}$ and $\omega$-dependent transport coefficients due to nonlinear fluctuations. However, the procedure of decoupling is known to be effective in the limit $k \rightarrow 0$ only, when the equilibrium solution of FPE could be expressed in a gaussian form. On the other hand, it is obvious that the mode-coupling theory should give us correct results, at least in a hydrodynamic region of $\boldsymbol{k}$. Some attempts have been made $[4,5,17]$ to determine the upper limit of $\boldsymbol{k}$, where the mode-coupling theory still holds. In [17] the authors suggested that, taking into account kinetic processes, one can improve the description and the mode-coupling theory gives reliable results up to the values of dimensionless $k^{*}=1 \div 3$. In the present paper we propose another explanation of the mentioned results which takes into consideration nonlocal thermodynamic relations (which is of particular importance in the vicinity of the critical point $[16])$.

The paper is organized in the following way. In section 2 we write down FPE for a distribution function of coarse-grained dynamic variables on the basis of a nonequilibrium statistical operator. Section 3 is devoted to the calculation of thermodynamic forces and hydrodynamic velocities in both the gaussian and higher order approximations in fluctuations. In section 4 we obtain the expressions for generalized ( $\boldsymbol{k}$ - and $\omega$-dependent) transport coefficients using the mode-coupling theory. The subject of section 5 is calculation of the dynamic structure factor of a simple liquid on the basis of the results obtained in the previous sections. A few words will be said about the advantages of nonlocal thermodynamic relations and approximations for triple static correlation functions joining the irreducible parts of hydrodynamic velocities and quadratic fluctuations of basic variables. One should point out that fluctuations of the total energy will be taken into account, while in the majority of cases a viscoelastic approach has been used $[4,5,10]$. An analysis of the sound mode is performed and the coefficient at $k^{5 / 2}$ in the expression for a sound frequency dispersion is calculated. A comparison with the experiment and computer simulation will be provided. Some conclusions are drawn in the last section. 


\section{Fokker-Planck equation for the distribution function of coarse-grained dynamic variables}

We are studying a one-component classical liquid, where hydrodynamic fluctuations can be described by a set of orthogonal dynamical variables $\hat{a}_{m \boldsymbol{k}}$ : Fouriertransforms of the number density fluctuation $\hat{n}_{\boldsymbol{k}}$, the momentum density fluctuation $\hat{j}_{\boldsymbol{k}}$ and the enthalpy density fluctuation $\hat{h}_{\boldsymbol{k}}$

$$
\begin{gathered}
\hat{n}_{\boldsymbol{k}}=\frac{1}{\sqrt{N}}\left(\sum_{j=1}^{N} \mathrm{e}^{-\mathrm{i} \boldsymbol{k} \boldsymbol{r}_{j}}-\langle\hat{N}\rangle_{0} \delta_{k 0}\right), \quad \hat{\boldsymbol{j}}_{\boldsymbol{k}}=\frac{1}{\sqrt{N}}\left(\sum_{j=1}^{N} \boldsymbol{p}_{j} \mathrm{e}^{-\mathrm{i} \boldsymbol{k} \boldsymbol{r}_{j}}-\langle\hat{\boldsymbol{P}}\rangle_{0} \delta_{k 0}\right), \\
\hat{h}_{\boldsymbol{k}}=\hat{\varepsilon}_{\boldsymbol{k}}-\left(\hat{\varepsilon}_{\boldsymbol{k}}, \hat{n}_{-\boldsymbol{k}}\right)\left(\hat{n}_{\boldsymbol{k}}, \hat{n}_{-\boldsymbol{k}}\right)^{-1} \hat{n}_{\boldsymbol{k}}
\end{gathered}
$$

where $\hat{\varepsilon}_{\boldsymbol{k}}$ is the Fourier-transform of the total energy density fluctuations

$$
\hat{\varepsilon}_{\boldsymbol{k}}=\frac{1}{\sqrt{N}}\left(\sum_{j=1}^{N}\left[\frac{\boldsymbol{p}_{j}^{2}}{2 m}+\frac{1}{2} \sum_{j \neq l=1}^{N} \Phi\left(\left|\boldsymbol{r}_{j l}\right|\right)\right] \mathrm{e}^{-\mathrm{i} \boldsymbol{k} \boldsymbol{r}_{j}}-\langle\hat{E}\rangle_{0} \delta_{k 0}\right),
$$

$\boldsymbol{p}_{j}, m$ correspond to the momentum and the mass of the particle, $\hat{N}$ denotes the total particle number, $\hat{\boldsymbol{P}}$ means the total momentum of the system and $\hat{E}-$ the total energy of the system. $\Phi\left(\left|\boldsymbol{r}_{j l}\right|\right)$ is the potential energy which we choose in the form of the Lennard-Jones potential $\Phi_{\mathrm{LJ}}(r)=4 \epsilon_{\mathrm{LJ}}\left[\left(\sigma_{\mathrm{LJ}} / r\right)^{12}-\left(\sigma_{\mathrm{LJ}} / r\right)^{6}\right]$ (let us introduce effective correlation time $\tau_{\sigma}=\sigma_{\mathrm{LJ}}\left(\mathrm{m} / \varepsilon_{\mathrm{LJ}}\right)^{1 / 2}$ as well $) ; \delta_{i j}$ denotes the Kronecker delta-symbol. The values of a wave-vector in (1) are restricted from above to $k_{0}$, that determines hydrodynamic scales $1 / k_{0} ;\left(\hat{a}_{\boldsymbol{k}}, \hat{b}_{-\boldsymbol{k}}\right) \equiv\left\langle\hat{a}_{\boldsymbol{k}} \hat{b}_{-\boldsymbol{k}}\right\rangle_{0}$ and $\langle\cdots\rangle_{0}=\int \mathrm{d} \Gamma_{N} \cdots \rho_{0}\left(x^{N}\right)$, where $\rho_{0}\left(x^{N}\right)$ means the equilibrium distribution function, $\mathrm{d} \Gamma_{N}=(\mathrm{d} x)^{N} / N$ !; $\mathrm{d} x=\mathrm{d} \boldsymbol{p}_{i} \mathrm{~d} \boldsymbol{r}_{i}$.

Dynamic variables (1)-(2) obey the conservation laws in the form

$$
\mathrm{i} L_{N} \hat{a}_{n \boldsymbol{k}} \equiv \dot{\hat{a}}_{n \boldsymbol{k}}=-\mathrm{i} \boldsymbol{k} \hat{\boldsymbol{I}}_{n \boldsymbol{k}},
$$

where $\mathrm{i} L_{N}$ denotes the Liouville operator, and expressions for the corresponding microscopic fluxes $\hat{\boldsymbol{I}}_{n \boldsymbol{k}}$ could be written down explicitly.

Complete information about hydrodynamic fluctuations is contained in the nonequilibrium distribution function [14] of dynamic variables (1):

$$
f(a ; t)=\langle\delta(\hat{a}-a)\rangle^{t}, \quad \delta(\hat{a}-a)=\prod_{n, \boldsymbol{k}} \delta\left(\hat{a}_{n \boldsymbol{k}}-a_{n \boldsymbol{k}}\right)
$$

where $\langle\cdots\rangle^{t}=\int \mathrm{d} \Gamma_{N} \cdots \rho\left(x^{N} ; t\right)$ denotes an averaging with the nonequilibrium statistical operator $\rho\left(x^{N} ; t\right)[18]$, obeying the Liouville equation:

$$
\frac{\partial}{\partial t} \rho\left(x^{N} ; t\right)+\mathrm{i} L_{N} \rho\left(x^{N} ; t\right)=0 .
$$


Taking into account the definition of (4) and (5) one can pass to FPE for the nonequilibrium distribution function $f(a ; t)$ which, by using the Markovian approximation and restriction by the second derivatives with respect to hydrodynamic variables, can be presented in the following form [14]:

$$
\begin{aligned}
\frac{\partial}{\partial t} f(a ; t) & +\sum_{\boldsymbol{k}, m, n} \frac{\partial}{\partial a_{n \boldsymbol{k}}}\left(v_{n \boldsymbol{k}}(a)+D_{n m}(\boldsymbol{k} ; a) F_{m}(\boldsymbol{k} ; a)\right) f(a ; t) \\
& -\sum_{\boldsymbol{k}, m, n} \frac{\partial}{\partial a_{n \boldsymbol{k}}} D_{n m}(\boldsymbol{k} ; a) \frac{\partial}{\partial a_{m-\boldsymbol{k}}} f(a ; t)=0
\end{aligned}
$$

where

$$
v_{n \boldsymbol{k}}(a)=\left\langle\mathrm{i} L_{N} \hat{a}_{n \boldsymbol{k}}\right\rangle_{a}, \quad\langle\cdots\rangle_{a}=\int \mathrm{d} \Gamma_{N} \cdots \delta(\hat{a}-a) / W(a)
$$

denote hydrodynamic velocities,

$$
W(a)=\int \mathrm{d} \Gamma_{N} \delta(\hat{a}-a)
$$

means statistical weight of the state with fixed values of fluctuations that determines entropy $S(a)=\ln W(a)$, and thermodynamic forces $F_{n}(\boldsymbol{k} ; a)$

$$
F_{n}(\boldsymbol{k} ; a)=\frac{1}{W(a)} \frac{\partial}{\partial a_{n \boldsymbol{k}}} W(a)=\frac{\partial}{\partial a_{n \boldsymbol{k}}} S(a),
$$

are conjugated to $a_{n \boldsymbol{k}}$,

$$
D_{n m}(\boldsymbol{k} ; a)=\int_{-\infty}^{0} \mathrm{~d} t \mathrm{e}^{\varepsilon t}\left\langle\left(1-\wp_{a}\right) \mathrm{i} L_{N} \hat{a}_{n \boldsymbol{k}} T_{a}(t)\left(1-\wp_{a}\right) \mathrm{i} L_{N} \hat{a}_{m-\boldsymbol{k}}\right\rangle_{a}
$$

denote generalized kinetic coefficients. Here $T_{a}\left(t, t^{\prime}\right)=\exp \left\{\left(t-t^{\prime}\right)\left(1-\wp_{a}\right) \mathrm{i} L_{N}\right\}$ means a "truncated" evolution operator [14], while $\wp_{a}$ denotes a projection operator on the state with fixed $\hat{a}=a$.

From the structure of expressions (7)-(9) it is easy to see that the calculation of thermodynamic forces means the evaluation of statistical weight $W(a)$. As a rule, $W(a)$ is approximated by a gaussian form of the set $a_{n k}$. However, one has to calculate nongaussian terms in $v_{n \boldsymbol{k}}(a)$ to be able to determine generalized transport coefficients. It is the subject of the next section where terms quadratic in fluctuations will be obtained on the basis of the cumulant expansion $[6,19,28]$ of the statistical weight for a state with fixed values of $a_{n k}$.

\section{Calculation of thermodynamic forces and hydrodynamic velocities}

At first we shall calculate thermodynamic forces $F_{n \boldsymbol{k}}$. For this purpose we use an integral representation of the $\delta$-function:

$$
\delta\left(\hat{a}_{n \boldsymbol{k}}-a_{n \boldsymbol{k}}\right)=\int \mathrm{d} \omega \exp \left\{-\mathrm{i} \pi \omega_{n \boldsymbol{k}}\left(\hat{a}_{n \boldsymbol{k}}-a_{n \boldsymbol{k}}\right)\right\} .
$$


Then, performing the cumulant expansion $[6,19,28]$, we can write down expressions for $F_{n \boldsymbol{k}}$ up to the second order in fluctuations:

$$
F_{m}(\boldsymbol{k} ; a)=F_{m}^{\mathrm{G}}(\boldsymbol{k} ; a)+\sum_{r, l} \sum_{\boldsymbol{k}^{\prime}} U_{m r l}\left(\boldsymbol{k}, \boldsymbol{k}^{\prime}\right) a_{r \boldsymbol{k}^{\prime}} a_{l \boldsymbol{k}-\boldsymbol{k}^{\prime}},
$$

where we have used the following denotations for gaussian contribution $F_{m}^{\mathrm{G}}(\boldsymbol{k} ; a)$ and tensor $U_{m r l}\left(\boldsymbol{k}, \boldsymbol{k}^{\prime}\right)$ :

$$
\begin{gathered}
F_{m}^{\mathrm{G}}(\boldsymbol{k} ; a)=-\frac{a_{m \boldsymbol{k}}}{\left\langle\hat{a}_{m \boldsymbol{k}} \hat{a}_{m-\boldsymbol{k}}\right\rangle_{E}}, \\
U_{m r l}\left(\boldsymbol{k}, \boldsymbol{k}^{\prime}\right)=\frac{M_{3}^{m r l}\left(\boldsymbol{k}, \boldsymbol{k}^{\prime}\right)}{M_{2}^{m m}(\boldsymbol{k}) M_{2}^{r r}\left(\boldsymbol{k}^{\prime}\right) M_{2}^{l l}\left(\boldsymbol{k}-\boldsymbol{k}^{\prime}\right)} .
\end{gathered}
$$

Here $\langle\ldots\rangle_{E}=W(E)^{-1} \int \ldots \mathrm{d} \Gamma_{N}, W(E)=\int \mathrm{d} \Gamma_{N}$ under the condition $\varepsilon_{k=0}=$ $E=$ const;

$$
M_{n}^{m_{1} \cdots m_{n}}\left(\boldsymbol{k}_{1}, \cdots, \boldsymbol{k}_{n}\right)=\left\langle\hat{a}_{m_{1} \boldsymbol{k}_{1}} \cdots a_{m_{n} \boldsymbol{k}_{n}}\right\rangle_{E}^{\mathrm{c}}, \quad\left(\boldsymbol{k}_{1}+\ldots+\boldsymbol{k}_{n}=0\right)
$$

denotes $n$-th cumulant (index $c$ means cumulant averaging).

In a similar way we can calculate hydrodynamic velocities $v_{m k}(a)(7)$. For this purpose let us introduce the parametrical statistical weight as

$$
W(a, \lambda)=\int \mathrm{d} \Gamma_{N} \exp \left\{\sum_{\boldsymbol{k}, \lambda} \lambda_{m \boldsymbol{k}} \dot{\hat{a}}_{m \boldsymbol{k}}\right\} \delta(\hat{a}-a), v_{m \boldsymbol{k}}(a)=\left.\frac{\partial \ln W(a, \lambda)}{\partial \lambda_{m \boldsymbol{k}}}\right|_{\lambda_{m \boldsymbol{k}}=0} .
$$

Now, carrying out the cumulant expansion like that in the case of $W(a)$, we can write down expressions for $v_{r k}$ :

$$
v_{r \boldsymbol{k}}(a)=v_{r \boldsymbol{k}}^{\mathrm{G}}(a)+\sum_{i, l} \sum_{\boldsymbol{k}^{\prime}} V_{r i l}\left(\boldsymbol{k}, \boldsymbol{k}^{\prime}\right) a_{i \boldsymbol{k}^{\prime}} a_{l \boldsymbol{k}-\boldsymbol{k}^{\prime}} \equiv v_{r \boldsymbol{k}}^{\mathrm{G}}(a)+v_{r \boldsymbol{k}}^{i r}(a)
$$

where we have for gaussian contribution $v_{r \boldsymbol{k}}^{\mathrm{G}}(a)$

$$
v_{r \boldsymbol{k}}^{\mathrm{G}}(a)=\sum_{l} \mathrm{i} \Omega_{r l}(\boldsymbol{k}) a_{l \boldsymbol{k}}, \quad \mathrm{i} \Omega_{r l}(\boldsymbol{k})=\frac{\left\langle\dot{\hat{a}}_{r \boldsymbol{k}} \hat{a}_{l-\boldsymbol{k}}\right\rangle_{E}}{\left\langle\hat{a}_{l \boldsymbol{k}} \hat{a}_{l-\boldsymbol{k}}\right\rangle_{E}},
$$

and for tensor $V_{\text {ril }}\left(\boldsymbol{k}, \boldsymbol{k}^{\prime}\right)$ :

$$
\begin{aligned}
V_{r i l}\left(\boldsymbol{k}, \boldsymbol{k}^{\prime}\right)= & \left(\frac{\left\langle\dot{\hat{a}}_{r \boldsymbol{k}} \hat{a}_{i \boldsymbol{k}^{\prime}} \hat{a}_{l \boldsymbol{k}-\boldsymbol{k}^{\prime}}\right\rangle_{E}}{\left\langle\hat{a}_{i \boldsymbol{k}^{\prime}} \hat{a}_{i-\boldsymbol{k}^{\prime}}\right\rangle_{E}\left\langle\hat{a}_{l \boldsymbol{k}-\boldsymbol{k}^{\prime}} \hat{a}_{l \boldsymbol{k}^{\prime}-\boldsymbol{k}}\right\rangle_{E}}\right. \\
& \left.-\sum_{s} \mathrm{i} \Omega_{r s}(\boldsymbol{k}) \frac{\left\langle\hat{a}_{s \boldsymbol{k}} \hat{a}_{i \boldsymbol{k}^{\prime}} \hat{a}_{l \boldsymbol{k}-\boldsymbol{k}^{\prime}}\right\rangle_{E}}{\left\langle\hat{a}_{s \boldsymbol{k}} \hat{a}_{s-\boldsymbol{k}}\right\rangle_{E}\left\langle\hat{a}_{i \boldsymbol{k}^{\prime}} \hat{a}_{i-\boldsymbol{k}^{\prime}}\right\rangle_{E}\left\langle\hat{a}_{l \boldsymbol{k}-\boldsymbol{k}^{\prime}} \hat{a}_{l \boldsymbol{k}^{\prime}-\boldsymbol{k}}\right\rangle_{E}}\right) .
\end{aligned}
$$

It is interesting to compare expression (17)-(19) with the results of the projection on the orthonormalized basis of higher fluctuations [20]. One can see that the presented results differ from [20] only by the normalization factor in denominators of 
(19). The second observation that could be done from (10) and (18) is an exact Green-Kubo representation for kinetic coefficients in the gaussian approximation for hydrodynamic velocities with projection operators coinciding with Mori projectors as it should be in the linear hydrodynamics case. An explicit form of the next term of the projecting operator $\wp_{a}$ could be extracted from (19) and, as it has already been said, this form resembles a projection operator on the higher fluctuations basis [20].

From the point of view of hydrodynamic velocities one can conclude that kinetic coefficients (10) can be represented as a sum of the gaussian term and the term due to nonlinear fluctuations:

$$
D_{m n}(\boldsymbol{k} ; a)=D_{m n}^{\mathrm{G}}(\boldsymbol{k} ; a)+\bar{D}_{m n}(\boldsymbol{k} ; a) .
$$

It is the second term in (20) that determines time-spatial dispersion of the transport coefficients. In the next section we shall obtain the expressions for generalized transport coefficients within a mode-coupling approach using the approximation quadratic in fluctuations of hydrodynamic variables.

\section{Calculation of time-spatial dispersion of transport coefficients}

At the beginning let us introduce the time correlation functions (TCF) of collective variables (1):

$$
\left(\hat{a}_{m \boldsymbol{k}}(t), \hat{a}_{n-\boldsymbol{k}}\right) \equiv\left(\exp \{\mathrm{i} L t\} \hat{a}_{m \boldsymbol{k}}, \hat{a}_{n-\boldsymbol{k}}\right)
$$

where time evolution is determined by the Liouville operator and averaging is performed over equilibrium distribution. For the Fourier-transform of TCF (21) one can obtain the following matrix equation [23]:

$$
G(\boldsymbol{k}, \omega)=(-\mathrm{i} \omega+\mathrm{i} \Omega(\boldsymbol{k})+\Gamma(\boldsymbol{k}, \omega))^{-1}\left(\hat{a}_{\boldsymbol{k}}, \hat{a}_{-\boldsymbol{k}}\right),
$$

where $\Gamma(\boldsymbol{k}, \omega)=D(\boldsymbol{k}, \omega)\left(\hat{a}_{\boldsymbol{k}}, \hat{a}_{-\boldsymbol{k}}\right)^{-1}$ and kinetic coefficients $D_{m n}(\boldsymbol{k}, \omega)$ can be calculated using the mode-coupling formalism $[2,3,11,16]$, as follows:

$$
\begin{aligned}
D_{m n}(\boldsymbol{k}, \omega)= & D_{m n}^{0}(\boldsymbol{k})+\sum_{\boldsymbol{k}^{\prime}} V_{m l s}\left(\boldsymbol{k}, \boldsymbol{k}^{\prime}\right) V_{n l^{\prime} s^{\prime}}\left(\boldsymbol{k}, \boldsymbol{k}^{\prime}\right) \int_{-\infty}^{\infty} \frac{\mathrm{d} \omega^{\prime}}{\pi}\left[G_{l l^{\prime}}\left(\boldsymbol{k}^{\prime}, \omega^{\prime}\right)\right. \\
& \left.\times G_{s s^{\prime}}\left(\boldsymbol{k}-\boldsymbol{k}^{\prime}, \omega-\omega^{\prime}\right)+G_{l s^{\prime}}\left(\boldsymbol{k}^{\prime}, \omega^{\prime}\right) G_{l^{\prime} s}\left(\boldsymbol{k}-\boldsymbol{k}^{\prime}, \omega-\omega^{\prime}\right)\right] .
\end{aligned}
$$

(Summation over repeated indexes in (23) is assumed). The first term in (23) denotes a "bare" transport coefficient determined as

$$
D_{m n}^{0}(\boldsymbol{k})=\int \mathrm{d} a f_{0}(a) D_{m n}(\boldsymbol{k} ; a),
$$

where averaging is performed with an equilibrium solution to FPE (6). 
In fact, relations (22)-(23) form a coupled chain of nonlinear integral equations for TCF where generalized kinetic coefficients $\Gamma(\boldsymbol{k}, \omega)$, in theirs turn, are expressed via the corresponding TCF. This chain of equations can be solved in the lowest orders in $k$ by successive iterations [3], taking for the zeroth approximation for TCF their linear hydrodynamic expressions and restricting oneself by thermodynamic values $V_{m l s}\left(\boldsymbol{k}, \boldsymbol{k}^{\prime}\right) \approx V_{m l s}(0,0)$ for triple static correlation functions (TrSCF):

$$
\begin{array}{r}
D_{m n}^{(1)}(\boldsymbol{k}, \omega)=D_{m n}^{0}(\boldsymbol{k})+\mathrm{i} \boldsymbol{k}^{2} \sum_{\alpha, \beta l, l^{\prime}, s, s^{\prime}} \sum_{\boldsymbol{k}^{\prime}} V_{m l s}^{0}(0) V_{n l^{\prime} s^{\prime}}^{0}(0) \\
\times \frac{A_{\alpha}^{l l^{\prime}}(0) A_{\beta}^{s s^{\prime}}(0)+A_{\alpha}^{l s^{\prime}}(0) A_{\beta}^{s l^{\prime}}(0)}{\omega+\mathrm{i}\left[\omega_{\alpha}\left(\boldsymbol{k}-\boldsymbol{k}^{\prime}\right)+\omega_{\beta}\left(\boldsymbol{k}^{\prime}\right)\right]}
\end{array}
$$

where

$$
V_{m i j}^{0}\left(\boldsymbol{k}^{\prime}\right)=\frac{\left\langle\hat{\boldsymbol{I}}_{m k=0} \hat{a}_{i \boldsymbol{k}^{\prime}} \hat{a}_{j-\boldsymbol{k}^{\prime}}\right\rangle_{E}}{\left\langle\hat{a}_{i \boldsymbol{k}^{\prime}} \hat{a}_{i-\boldsymbol{k}^{\prime}}\right\rangle_{E}\left\langle\hat{a}_{j \boldsymbol{k}^{\prime}} \hat{a}_{j-\boldsymbol{k}^{\prime}}\right\rangle_{E}}-\sum_{l} \frac{\left\langle\hat{a}_{l k=0} \hat{a}_{i \boldsymbol{k}^{\prime}} \hat{a}_{j-\boldsymbol{k}^{\prime}}\right\rangle_{E}\left\langle\hat{\boldsymbol{I}}_{m k=0} \hat{a}_{l k=0}\right\rangle_{E}}{\left\langle\hat{a}_{l k=0} \hat{a}_{l k=0}\right\rangle_{E}\left\langle\hat{a}_{i \boldsymbol{k}^{\prime}} \hat{a}_{i-\boldsymbol{k}^{\prime}}\right\rangle_{E}\left\langle\hat{a}_{j \boldsymbol{k}^{\prime}} \hat{a}_{j-\boldsymbol{k}^{\prime}}\right\rangle_{E}} .
$$

In $(25), \omega_{\alpha}(\boldsymbol{k})$ are eigenvalues, $A_{\alpha}^{l l^{\prime}}(\boldsymbol{k})=\sum_{\alpha} X_{l \alpha}(\boldsymbol{k}) X(\boldsymbol{k})_{l^{\prime} \alpha}^{-1}\left(\hat{a}_{\boldsymbol{k}}, \hat{a}_{-\boldsymbol{k}}\right)_{l l^{\prime}}, X_{l \alpha}(\boldsymbol{k})$ are eigenvectors of the $T(\boldsymbol{k})$-matrix for TCF of linear hydrodynamics in the Markovian approximation [24] and $\alpha$-, $\beta$ - summation is carried out over all hydrodynamic modes: sound modes $\omega_{1,2}(\boldsymbol{k})= \pm \mathrm{i} c k+\gamma \boldsymbol{k}^{2},(c$ is sound velocity, $\gamma$ means a sound damping constant) and heat mode $\omega_{3}(\boldsymbol{k})=D_{\mathrm{T}} \boldsymbol{k}^{2}\left(D_{\mathrm{T}}\right.$ denotes a heat transfer constant) as well as transversal mode connected with shear diffusion $\omega_{\perp}(\boldsymbol{k})=$ $D_{\perp} \boldsymbol{k}^{2}$. The second step is to insert the value of $D_{m n}^{(1)}(\boldsymbol{k}, \omega)$ in (22) which gives us the first approximation for TCF. Performing successive iterations in (22)-(23) yields the appearance of long time tails in TCF $[3,11,12]$ due to nonanalytic behaviour of transport coefficients in $\omega$. On the other hand, nonanalytic dependance in $k$ leads to renormalization of the corresponding modes. For instance, one can see that dispersion of a sound mode might be presented in the following form:

$$
\omega_{s}(k)= \pm \mathrm{i} c k+\gamma k^{2}+\sum_{n=1}^{\infty} \delta_{s}(n) k^{3-2^{-n}},
$$

where functions $\delta_{s}(n)$ are connected with amplitudes $V_{m n l}^{0}(0)$. The last equation gives the well-known $k^{5 / 2}$ correction to the sound mode in the lowest order in wavenumber. It was shown $[3,16,20]$, that taking into consideration either the cubic fluctuation or nonlocal values $V_{n n l}^{0}\left(\boldsymbol{k}^{\prime}\right)$ of amplitudes give corrections of higher orders in $k$ and $\omega$ in comparison with the iteration procedure and is not taken into account when $k$ is small. However, their contributions become important in the intermediate domain of wave-number where the mode-coupling theory is believed to be still reliable [17]. It is obvious that consideration of cubic fluctuations needs the knowledge of the 4th order static correlation functions. Instead, we restrict ourselves by taking into account nonlocalities in functions (26).

It should be emphasised that our approach is consistent with those of [12] giving at the beginning the appearance of $\sqrt{k}$-contributions to the dispersion of transport 
coefficients for frequencies much lower than the diffusive ones and, afterwards, square root frequency dependence $\sqrt{\omega}$ for an intermediate frequency region. In this context we have a picture resembling the results of [25] where a double crossover in frequency dependence of shear viscosity when passing from regular to nonanalytic and again to regular behaviour at high $\omega$ was observed in the case of nonzero wave-numbers. However, we have not introduced specific features of transport coefficients at small time scales (like that dealt with cage effects in [25]), so we cannot claim for a proper description of high frequency behaviour of transport coefficients. Nevertheless, our "long tail" time features of transport coefficients have correct prefactors while the result for viscosity in [5] is not correct.

We left aside the convergency problem of the corresponding series of the iterative procedure. As far as we know, it still remains an open question for dense fluids [2,3]. It can be pointed out that another problem stressed in the cited papers, which deals with divergency of Burnett and super-Burnett terms, turned out to be closed $[11,12]$, so that formal expansion of the sound mode contribution to the transport coefficient in the series of $k$ is not correct, when $c k t \rightarrow \infty$.

At the end of the present section we would like to write down the relations between kinetic coefficients $\Gamma_{m n}(\boldsymbol{k}, \omega)$ and nonlocal transport coefficients:

$$
\eta_{\boldsymbol{k} \omega}=\eta^{0}+\frac{n m}{2 \boldsymbol{k}^{2}} \Gamma_{j_{\alpha} j_{\beta}}(\boldsymbol{k}, \omega)\left(\delta_{\alpha \beta}-n_{\alpha} n_{\beta}\right)
$$

for generalized shear viscosity $\eta_{\boldsymbol{k} \omega}$, where $n$ denotes the number density;

$$
\frac{4}{3} \eta_{\boldsymbol{k} \omega}+\zeta_{\boldsymbol{k} \omega}=\frac{4}{3} \eta^{0}+\zeta^{0}+\frac{n m}{\boldsymbol{k}^{2}} n_{\alpha} n_{\beta} \Gamma_{j_{\alpha} j_{\beta}}(\boldsymbol{k}, \omega)
$$

for generalized longitudinal viscosity, where $\zeta_{\boldsymbol{k} \omega}$ denotes the generalized bulk viscosity;

$$
\lambda_{\boldsymbol{k} \omega}=\lambda^{0}+\frac{n C_{v}}{\boldsymbol{k}^{2}} \Gamma_{h h}(\boldsymbol{k}, \omega)
$$

for generalized thermal conductivity $\lambda_{\boldsymbol{k} \omega}$, where $C_{v}$ means specific heat at the constant volume;

$$
\xi_{\boldsymbol{k} \omega}=\frac{n C_{v}}{\boldsymbol{k}^{2}} \Gamma_{j_{\alpha} h}(\boldsymbol{k}, \omega) n_{\alpha}
$$

for generalized thermoviscous coefficient $\xi_{\boldsymbol{k}}$ vanishing in the limit $k \rightarrow 0$. In expressions (28)-(31), superscripts (0) are used for "bare" values of the corresponding transport coefficients, $n_{\alpha}=k_{\alpha} / k$.

In the next section we shall calculate a dynamic structure factor for the Lennard-Jones fluid both in local and nonlocal thermodynamics approximations in the intermediate region of dimensionless parameter $k^{*}=k \sigma_{\mathrm{LJ}}$.

\section{Calculation of a dynamic structure factor. Investigation of sound mode dispersion}

For the calculation of transport coefficients dispersion we start from equation (25). Passing from sum to integral $\sum_{\boldsymbol{k}^{\prime}} \ldots \rightarrow \frac{1}{(2 \pi)^{3}} \int \ldots \mathrm{d} \boldsymbol{k}^{\prime}$ and performing 
regularization of the corresponding integral [11], which means a substitution of "observable" values of transport coefficients at $k=0, \omega=0$ for "bare" ones, we obtain:

$$
D_{m n}^{(1)}(\boldsymbol{k}, \omega)=D_{m n}(0,0)+\int \frac{\mathrm{d} \boldsymbol{k}^{\prime}}{(2 \pi)^{3}} \int_{-\infty}^{\infty} \frac{\mathrm{d} \omega^{\prime}}{2 \pi}\left[M_{m n}\left(\boldsymbol{k}, \omega ; \boldsymbol{k}^{\prime}, \omega^{\prime}\right)-M_{m n}\left(0,0 ; \boldsymbol{k}^{\prime}, \omega^{\prime}\right)\right],
$$

where

$$
\begin{aligned}
& M_{m n}\left(\boldsymbol{k}, \omega ; \boldsymbol{k}^{\prime}, \omega^{\prime}\right)=\boldsymbol{k}^{2} \sum_{l, s, l^{\prime}, s^{\prime}} V_{m l s}^{0}(0) V_{n l^{\prime} s^{\prime}}^{0}(0) \\
& \quad \times\left(G_{l l^{\prime}}^{0}\left(\boldsymbol{k}^{\prime}, \omega^{\prime}\right) G_{s s^{\prime}}^{0}\left(\boldsymbol{k}-\boldsymbol{k}^{\prime}, \omega-\omega^{\prime}\right)+G_{l s^{\prime}}^{0}\left(\boldsymbol{k}^{\prime}, \omega^{\prime}\right) G_{l^{\prime} s}^{0}\left(\boldsymbol{k}-\boldsymbol{k}^{\prime}, \omega-\omega^{\prime}\right)\right),
\end{aligned}
$$

and $G_{m n}^{0}(\boldsymbol{k}, \omega)$ means a zeroth (linear hydrodynamic) approximation for TCF. It should be pointed out that there is no ultraviolet divergency in integrals (33) in a three-dimensional case due to regularization [12]. In the dimensionalities $d=1$ and $d=2$, one has to solve the equation for transport coefficients by a self-consistent method $[21,22]$. Further, inserting (32) in (22) we come to a system of equations for TCF in the first approximation where the dispersion of transport coefficients due to nonlinear fluctuations has already been taken into account.

If one substitutes the values $V_{m l s}^{0}\left(\boldsymbol{k}^{\prime}\right)$ in (33) instead of $V_{m l s}^{0}(0)$, which means taking into account a nonlocal thermodynamic approximation, he will obtain nonanalytic contributions to transport coefficients of higher orders in wave-number and frequency. In this section we present contributions of both local and nonlocal thermodynamics to the dynamic structure factor of the Lennard-Jones fluid. The second approximation turned out to be more correct in comparison with molecular dynamics (MD) simulations (see figures 1 and 2).

Now let us turn our attention to triple static correlation functions (TrSCF) $V_{n l s}^{0}\left(\boldsymbol{k}^{\prime}\right)(26)$. It is well known that taking the limit $k^{\prime} \rightarrow 0$ in (26) one can express TrSCF via the corresponding thermodynamic relations $[3,11,16]$. It is the case of local thermodynamics, when pressure of the system at a given point is determined by the values of particle and internal energy densities at the same point. On the other hand, there are situations (for instance, in the vicinity of the phase transition point) when pressure at point $\boldsymbol{r}$ depends on characteristics of the liquid in the neighbouring points. It corresponds to the case when the entropy functional is determined not only via hydrodynamic variables but also through their derivatives. In [26] the authors showed that it follows from the necessity for FPE to have its equilibrium solution. Some interesting results were obtained in [27] when both microscopic phase density $\hat{n}(\boldsymbol{k}, \boldsymbol{p})=\sum_{j=1}^{N} \exp \left(-\mathrm{i} \boldsymbol{k} \boldsymbol{r}_{j}\right) \delta\left(\boldsymbol{p}-\boldsymbol{p}_{j}\right)$ and $\delta(\hat{a}-a)=\prod_{n, \boldsymbol{k}} \delta\left(\hat{a}_{n \boldsymbol{k}}-a_{n \boldsymbol{k}}\right)$ were taken into consideration. Unfortunately, the authors could not proceed to write general expressions for memory kernels only, though, taking into account the kinetic variable $\hat{n}(\boldsymbol{k}, \boldsymbol{p})$ gives a more realistic picture of the system behaviour. 
In the general case $k^{\prime} \neq 0$, the problem of an approximation for TrSCF arises. It means that we have to possess some information about distribution functions of higher orders. On the other hand, we use as input data the results for pair static correlation functions from MD. Having MD results for the pair static correlation functions $\left\langle\hat{\varepsilon}_{\boldsymbol{k}} \hat{n}_{-\boldsymbol{k}}\right\rangle_{0},\left\langle\hat{\varepsilon}_{\boldsymbol{k}} \hat{\varepsilon}_{-\boldsymbol{k}}\right\rangle_{0}$, one formally takes into account correlations of three and four particles, correspondingly. But it does not mean that we have expressions for 3- and 4-particle distribution functions; we observe only their "integral" effects. Hence, the problem consists in the approximation of TrSCF via pair ones. There are several approximations for higher distribution functions: convolution, different kinds of the Kirkwood superposition approximation [28]. In fact, all these approximations are of the zeroth order in parameter $n r_{\text {cor }}^{3}$, where $r_{\text {cor }}$ is an effective correlation radius which can be estimated by $\sigma_{\mathrm{LJ}}$ in the case of the Lennard-Jones liquid. In this context MD results for pair correlation functions could be treated as a certain "approbation" for distribution functions of higher orders. However, passing from distribution functions to pair correlation functions one can easily observe that various approximations have one common feature: bad long-wavelength behaviour. So far, as the main value of the integral in (32) is accumulated in the domain of small values of $k^{\prime}$, the "loss of precision" due to approximation for $\operatorname{TrSCF}(26)$ is inevitable. Hence, the problem is only to take consistently into account a greater number of interacting particles (because of high density of the system) when choosing a particular form for the higher-order distribution function.

In our calculation we use the convolution approximation giving the factorization

$$
S_{3}(\boldsymbol{k}, \boldsymbol{k}-\boldsymbol{q}, \boldsymbol{q}) \approx S_{2}(\boldsymbol{k}) S_{2}(\boldsymbol{q}) S_{2}(\boldsymbol{k}-\boldsymbol{q})
$$

for the triple static structure factor via pair ones. This approximation was shown to have better long-wavelength behaviour [29] than the superposition one. One can easily perform an inverse Fourier transformation in (34) from $\boldsymbol{k}$ - to $\boldsymbol{r}$-space, expressing triple distribution function $g_{3}\left(\left|\boldsymbol{r}_{2}-\boldsymbol{r}_{0}\right|,\left|\boldsymbol{r}_{1}-\boldsymbol{r}_{0}\right|,\left|\boldsymbol{r}_{1}-\boldsymbol{r}_{2}\right|\right)$ via pair ones as follows:

$$
\begin{aligned}
& g_{3}\left(\left|\boldsymbol{r}_{2}-\boldsymbol{r}_{0}\right|,\left|\boldsymbol{r}_{1}-\boldsymbol{r}_{0}\right|,\left|\boldsymbol{r}_{1}-\boldsymbol{r}_{2}\right|\right)=g_{2}\left(\left|\boldsymbol{r}_{2}-\boldsymbol{r}_{0}\right|\right) g_{2}\left(\left|\boldsymbol{r}_{1}-\boldsymbol{r}_{0}\right|\right)+g_{2}\left(\left|\boldsymbol{r}_{2}-\boldsymbol{r}_{1}\right|\right) g_{2}\left(\left|\boldsymbol{r}_{1}-\boldsymbol{r}_{0}\right|\right) \\
& \quad+g_{2}\left(\left|\boldsymbol{r}_{2}-\boldsymbol{r}_{0}\right|\right) g_{2}\left(\left|\boldsymbol{r}_{1}-\boldsymbol{r}_{2}\right|\right)+n \int \mathrm{d} \boldsymbol{r} g_{2}\left(\left|\boldsymbol{r}_{1}-\boldsymbol{r}_{2}-\boldsymbol{r}\right|\right) g_{2}\left(\left|\boldsymbol{r}_{0}-\boldsymbol{r}_{2}-\boldsymbol{r}\right| g_{2}(|\boldsymbol{r}|) . \quad\right. \text { (35) }
\end{aligned}
$$

Then, one can calculate TrSCF (26) in the approximation of triple correlation. Moreover, approximation (35) is found to give nice correspondence to the static correlation function $\left\langle\hat{n}_{\boldsymbol{k}} \hat{\varepsilon}_{-\boldsymbol{k}}\right\rangle_{0}$, calculated in MD.

The calculation of the dynamic structure factor has been performed for the same thermodynamic point as in paper [24]: for the reduced density $n^{*}=n \sigma_{\mathrm{LJ}}^{3}=$ 0.845 and reduced temperature $T^{*}=k_{\mathrm{B}} T / \varepsilon_{\mathrm{LJ}}=1.706$. All the pair static correlation functions encountered in (26) were taken from MD simulation of the cited paper as well as the values of transport coefficients at $k=0, \omega=0$. For TrSCF approximation (35) was made, while four-, five- and six-particle correlations were 


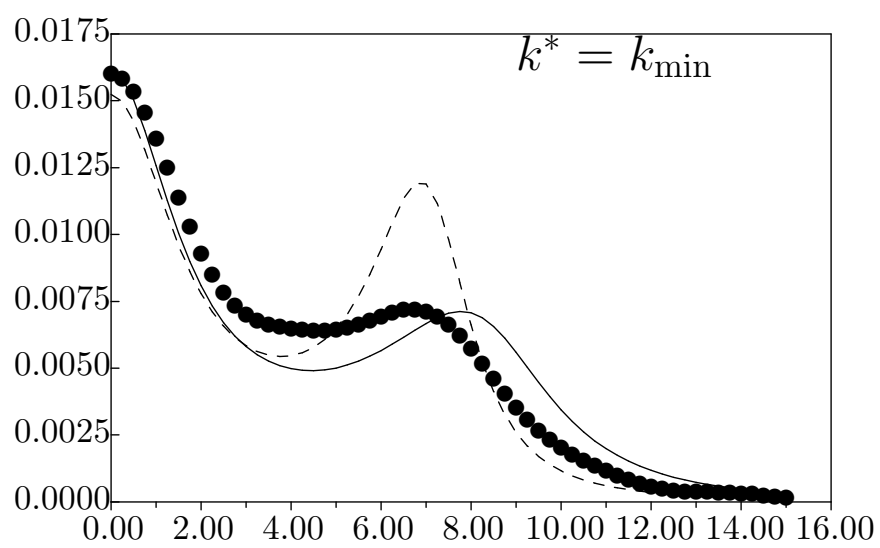

Figure 1. Dependence of dynamic structure factor $S(\boldsymbol{k}, \omega) \pi / \tau_{\sigma}$ on frequency $\omega^{*}=$ $\omega \tau_{\sigma}$ at $k^{*}=k_{\min } \cdot \bullet-\mathrm{MD}$ results; - - - -local thermodynamic approximation; - nonlocal thermodynamic approximation.

neglected. While solving a coupled chain of equations (22)-(23), three iterations were made in both local and nonlocal thermodynamic approximations.

The results for the dynamic structure factor are presented in figures 1 and 2 . One can see at $k^{*}=k_{\text {min }} \equiv 0.936\left(\right.$ where $\left.k^{*}=k \sigma_{\mathrm{LJ}}\right)$ that taking into consideration nonlocal thermodynamics effects (expressions for $V_{m n l}^{0}$ depend on $k$ ) gives much better results in comparison with the local thermodynamic approximation (when we put $V_{m n l}^{0}\left(k^{\prime}\right) \approx V_{m n l}^{0}(0)$ in $\left.(33)\right)$.

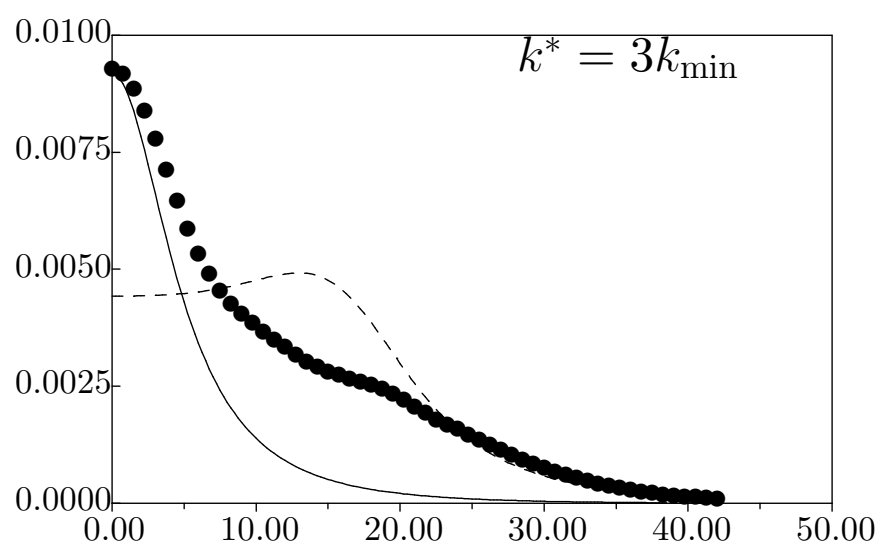

Figure 2. Dependence of dynamic structure factor $S(\boldsymbol{k}, \omega) \pi / \tau_{\sigma}$ on frequency $\omega^{*}=$ $\omega \tau_{\sigma}$ at $k^{*}=3 k_{\min } . \bullet-$ MD results; - - - -local thermodynamic approximation; - nonlocal thermodynamic approximation.

At $k^{*}=3 k_{\min }$ the local thermodynamic approximation does not describe a real picture at all. As to the nonlocal thermodynamic approximation, at least the form of the Rayleigh peak is partially reflected. However, one can see that at such a value of $k^{*}$, the mode-coupling theory does not have good correspondence to computer simulation and we are on the margin of the theory validity.

At small values of $k^{*}$ it is possible to restrict ourselves to the local thermodynamic approximation only as might be seen from figure 3, where a slight shift of the Brillouin peak of the dynamic structure factor due to nonlinear fluctuations 


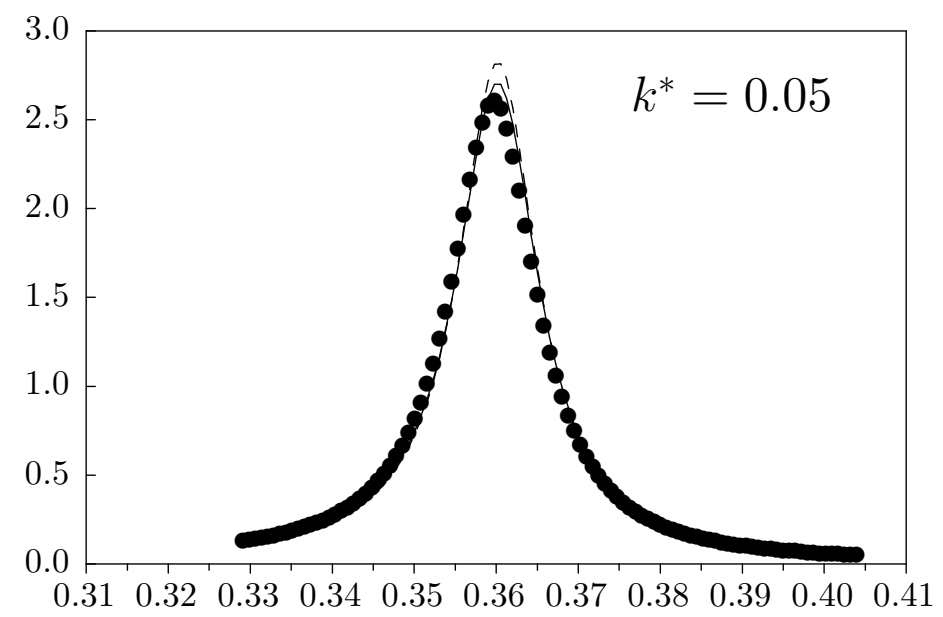

Figure 3. Brillouin peak position of dynamic structure factor $S(\boldsymbol{k}, \omega) \pi / \tau_{\sigma}$ as function of frequency $\omega^{*}=\omega \tau_{\sigma}$ at $k^{*}=0.05$. - -linear hydrodynamic results; - - - -local thermodynamic approximation; - - nonlocal thermodynamic approximation.

can be observed. Having calculated the values of transport coefficients at sound frequency $c k$, one can analyze the sound mode behaviour and compare this result with the molecular dynamics results [24] and the experiment data [17]. It allows us to calculate coefficient $a$ at a nonanalytical term in the expression for sound mode dispersion $\omega_{s}(k)= \pm \mathrm{i} c k+\gamma k^{2}+( \pm \mathrm{i}-1) a k^{5 / 2}$. Our calculation gives $a=0.92$ and the presented above dispersion law holds up to $k^{*} \sim 0.25$ when the necessity to take higher powers of $k^{*}$ into account appears. One has to point out that the obtained value of $a$ corresponds well to the result of [24], where it is calculated by least squares fitting and equals 1.46. But the problem mentioned above does not allow us to calculate TrSCF at small values of wave-vector and we face the problem similar to one appearing in MD simulation, when one has to extrapolate the results for static correlation functions in the domain $k \rightarrow 0$.

To a certain degree our results are comparative with the experimental data [17], where coefficient $a$ was equal to 0.90 , but our thermodynamic point slightly differs (in temperature) from that in the experiment.

\section{Concluding remarks}

The proposed approach allows one to carry out the calculation of time-spatial dispersion of transport coefficients and, consequently, to obtain the expressions for time correlation functions using the mode-coupling theory. We have calculated the dynamic structure factor of a simple liquid in both local and nonlocal thermodynamics approximations. The second approach appears to be more applicable in the case of intermediate values of wave-vector, while at low values of $k^{*}$ the local thermodynamics approximation is quite sufficient. From the mathematical point of view, taking into account $k$-dependence of $\operatorname{TrSCF}$, joining the irreducible parts of hydrodynamic velocities with quadratic fluctuations of collective variables 
means a consideration of terms of the next orders in wave-vector $\left(\sim k^{3 / 2}, k^{5 / 2}\right)$ in the expressions for dispersion of transport coefficients. In the domain of small wave-numbers these corrections are found to be of higher orders in comparison with the results of the iteration procedure for the solution of nonlinear integral equations and usually are not considered. However, these terms become important in the intermediate region of $k^{*}$ when we are on the margin of the validity of the mode-coupling theory. It is clear that one has to combine the iterative procedure with the consideration of nonlocal processes.

It should be emphasised that at $k^{*} \sim 1$ higher fluctuations (triple, etc.) give a certain contribution to expressions for transport coefficients and have to be taken into account too. On the other hand, even quadratic fluctuations give quite a reliable description of the system. For including the third-order fluctuations one has to know the fourth-order static correlation functions. It is clear that the approximations which should have been made to a considerable extent influence the final result and its validity cannot be considered to be better than in the case of quadratic fluctuations.

From the physical point of view, taking into account wave-dependence of TrSCF means, as has already been explained, a nonlocal thermodynamic approximation, when pressure at a given point of the liquid depends on characteristics in the neighbouring points. In this context it would be interesting to investigate the temperature dependence of the dynamic structure factor as soon as one is drawing near the critical point where the consideration of nonlocalities is essential.

The region of small $k^{*}$ is unreachable either by MD or by neutron scattering experiments. However, our calculations, performed at small $k^{*}$, allow us, firstly, to estimate the domain of validity of the mode-coupling theory and, secondly, to obtain numerical values at nonanalyticities of the corresponding transport coefficients.

\section{Acknowledgements}

The author is grateful to Prof. M.Tokarchuk, Dr. I.Mryglod for their permanent interest in the work and useful discussions and to Dr. I.Omelyan for the assistance in performing numerical calculations. I would like to acknowledge the financial support of the President of Ukraine too.

\section{References}

1. Rozmus W. Fluctuations in laser-produced plasmas. // Can. J. Phys., 1986, vol. 64, No. 8, p. 900-911.

2. Ernst N.H., Dorfman J.R. Nonanalytic dispersion relations for classical fluids. I. The dense gas. // Physica, 1972, vol. 61, p. 157.

3. Ernst N.H., Dorfman J.R. Nonanalytic dispersion relations for classical fluids. II. The general fluid. // Journ. of Stat. Phys., 1976, vol. 12, No. 4, p. 311-359. 
4. Munakata T., Igarashi A. Dynamic correlation functions of classical liquids. I. // Prog. of Theor. Phys., 1977, vol. 58, No. 6, p. 1345-1356.

5. Munakata T., Igarashi A. Dynamic correlation functions of classical liquids. II. // Prog. of Theor. Phys, 1978, vol. 60, No. 1, p. 45-59.

6. Ignatiuk V.V., Tokarchuk M.V. Statistical theory of nonlinear hydrodynamic fluctuations in ionic systems. // Teor. Mat. Fiz., 1996, vol. 108, No. 3, p. 1208-1221.

7. Zubarev D.N. Statistical thermodynamics of the turbulent transport processes. // Teor. Mat. Fiz., 1982, vol. 53, No. 1, p. 93-107.

8. Yukhnovskii I.R., Gurskii Z.A. Quantum Statistic Theory of Disordered Systems. Kiev, Naukova Dumka, 1991 (in Russian).

9. Morozov V.G. On fluctuational dispersion of transport coefficients in superfluid liquid. // Teor. Mat. Fiz., 1986, vol. 67, No. 1, p. 129-142.

10. Götze W. Liquid-glass Transitions. Moscow, Nauka, 1992 (in Russian).

11. Morozov V.G. DDr. Thesis, Institute of Mathematics of Acad. of Sci. of USSR (Moscow, 1987).

12. Morozov V.G. Nonlocal hydrodynamics and dispersion of transport coefficients in simple fluid. // Physica, 1983, vol. 117A, No. 3, p. 511-530.

13. Zubarev D.N. Two-times Green functions in statistical physics. // Uspekhi Phys. Nauk, 1960, vol. 71, No. 1, p. 71-116 (in Russian).

14. Zubarev D.N., Morozov V.G. Statistical mechanics of nonlinear hydrodynamic fluctuations. // Physica, 1983, vol. 120A, No. 3, p. 411-467.

15. Zubarev D.N., Khazanov A.D. Generalized Fokker-Planck equation and construction of projection operators for various methods of abbreviated description. // Teor. Mat. Fiz., 1978, vol. 34, No. 1, p. 69-80 (in Russian).

16. Kawasaki K. In: Phase Transitions and Critical Phenomena. New York, Academic, 1976, vol. 5a, p. 165.

17. de Schepper I.M., Verkerk P., Van Well A.A., de Graaf L.A. Non-analytic dispersion relations in liquid argon. // Physics letters A, 1984, vol. 104, No. 1, p. 29-32.

18. Zubarev D., Morozov V., Röpke G. Statistical Mechanics of Nonequilibrium Processes, Akademie Verlag, Berlin, 1996.

19. Idzyk I.M., Ignatyuk V.V., Tokarchuk M.V. Fokker-Planck equation for nonequilibrium distribution function of collective variables. I. Calculation of the statistical weight, entropy, hydrodynamic velocities. // Ukr. Fiz. Jurn., 1996, vol. 41, No. 56, p. 596-604 (in Ukrainian); II. Calculation of time-spatial dispersion of transport coefficients. // Ukr. Fiz. Jurn., 1996, vol. 41, No. 10, p. 1017-1021 (in Ukrainian).

20. Adzhemian L., Gringin A., Kuni F. Time asymptotics of the kinetic kernels of linear hydrodynamics. // Teor. Mat. Fiz., 1975, vol. 24. No. 2, p. 255-264 (in Russian).

21. Peletminskii S.V., Sokolovskii A.I. General equations of fluctuational hydrodynamics. // Ukr. Fiz. Jurn., 1992, vol. 37, No. 10, p. 1521.

22. Peletminskii S.V., Sokolovskii A.I. Spational dispersion of kinetic coefficients of fluid near equilibrium. // Ukr. Fis. Jurn., 1992, vol. 37, No. 11, p. 1702-1711.

23. Morozov V.G. Low-frequency correlation functions in case of nonlinear dynamics of fluctuations. // Physica A, 1982, vol. 110, No. 1, p. 201-221.

24. Mryglod I.M., Omelyan I.P., Tokarchuk M.V. Generalized collective modes for the Lennard-Jones fluid. // Mol. Phys., 1995, vol. 84, No. 2, p. 235-259.

25. Franosch T., Götze W. Mode-coupling theory for the shear viscosity in supercooled liquids. // Phys. Rev. E, 1998, vol. 57, No. 5, p. 5833-5840. 
26. Zubarev D.N., Morozov V.G. In: Problems of Modern Statistical Physics. Kiev, Naukova Dumka, 1985, p. 120 (in Russian).

27. Morozov V.G., Kobryn A.E., Tokarchuk M.V. Modified kinetic theory with consideration for slow hydrodynamical processes. // Cond. Matt. Phys., 1994, No. 4, p. 117-127.

28. Yukhnovskii I.R, Holovko M.F. Statistical Theory of Classical Equilibrium Systems. Singapore, World Scientific, 1987.

29. Jackson H.W., Feenberg E. // Rev. Mod. Phys., 1962, vol. 34, p. 686.

\title{
Розрахунок коефіцієнтів переносу та динамічного структурного фактора простих рідин
}

\author{
В.В.Ігнатюк \\ Інститут фізики конденсованих систем НАН України, \\ 290011 Львів, вул. Свєнціцького, 1 \\ Отримано 22 лютого 1999 року
}

На основі рівняння Фоккера-Планка для нерівноважної функції розподілу колективних змінних проводиться розрахунок динамічного структурного фактора та дисперсії коефіцієнтів переносу простих рідин. Для отримання замкнутого ланцюжка рівнянь для коефіцієнтів переносу, які $є$ функціями хвильового вектора $k$ та частоти $\omega$, застосовується формалізм теорії взаємодіючих мод. Показано, що в проміжній області $k$ співвідношення нелокальної термодинаміки дають точніші результати, тоді як при малих значеннях хвильового вектора можна обмежитись співвідношеннями локальної термодинаміки. Проведено дослідження звукової моди та обчислено коефіцієнт біля $k^{5 / 2}$ у виразі для дисперсії частоти звуку. Отримані результати порівнюються як з даними експерименту, так і молекулярної динаміки.

Ключові слова: рівняння Фоккера-Планка, часові кореляційні функції, нелінійні флуктуації, теорія взаємодіючих мод

PACS: 05.60. $+w, 05.70 . L n, 51.10 .+y, 61.20 . L c, 61.20 . \mathrm{Ne}$ 
